G U E S T E D I T O R I A L

\title{
Special Section on Biometrics: Advances in Security, Usability, and Interoperability
}

\author{
Claus Vielhauer* \\ Brandenburg University of Applied Sciences \\ Department of Informatics \& Media \\ Brandenburg an der Havel, 14770, Germany \\ E-mail: claus.vielhauer@fh-brandenburg.de \\ Berrin Yanikoğlu ${ }^{\dagger}$ \\ Sabanci University \\ Istanbul, Turkey \\ Sonia Garcia-Salicetti ${ }^{\dagger}$ \\ Institut National des Télécommunications \\ Evry, France \\ Richard M. Guest ${ }^{\dagger}$ \\ University of Kent \\ Canterbury, United Kingdom \\ Stephen J. Elliott ${ }^{\dagger}$ \\ Purdue University \\ West Lafayette, Indiana
}

Biometric user authentication systems are emerging from a highly specialized domain and are becoming part of the daily lives of many people all over the world. Until a few years ago, biometric techniques have been used almost exclusively in high-security areas for critical infrastructures or for convenience purposes such as login applications to personal computers. In these scenarios, biometrics has mainly involved small-scale, closed user group applications. In the research domain until recently, a main focus has been the elaboration of different methods for various active or passive biometric traits and their optimization toward high-recognition accuracy, and more recently toward multimodal biometrics.

While these areas remain relevant scientific domains, the tendency toward large-scale deployment of biometrics opens a multitude of additional re-

\footnotetext{
* Organizing guest editor.
}

${ }^{\dagger}$ Editorial board member. search challenges, which are of strong interest to the image processing community. For example, with many countries deciding to integrate digital images of biometric data in an interoperable manner in travel documents, we observe a step into a new dimension with respect to size, complexity, and also security requirements of biometric systems.

Challenges arise both from technical and nontechnical perspectives and include aspects ranging from security for biometric systems, integration in embedded and mobile devices, multiple expert systems and their performance evaluation, to interdisciplinary issues such as usability, privacy, user interface design for biometrics, as well as aging aspects.

This special section of JEl emphasizes original and novel contributions in the above-mentioned topics for biometric concepts, which are entirely or partly based on image processing, as well as security, integration, interoperability and evaluation aspects of single- and multiple-expert systems. It is composed of 17 peer-reviewed contributions from international researchers, which have been submitted partly by editorial invitation (10), as well as in response to an open call for papers (21). A major aspect of this special was to provide an actual overview of top-level research across those biometric subdomains that appear most interesting for the El community. Looking at the domains actually covered, today's biometric landscape appears well represented: 2-D and 3-D face modalities as well as fingerprint biometrics are addressed by the majority of papers (five and four papers, respectively). A third bias can be observed in the domain of handwriting analysis (two papers), whereas the remaining articles relate to multimodal/system aspects and the domains of iris, footprint, grip, and hand modalities.

In the first domain of research on face recognition, Yan, Osadciw, and Chen address the problem of appropriate feature selection and suggest a 
novel feature selection scheme. Their comparative experimental results show improvement over well-studied schemes such as principal component analysis and linear discriminant analysis. Also for the challenge of feature extraction for face biometrics, Štruc et al. suggest a hybrid method based on trace transform and kernel partial-leastsquares discriminant analysis and report better recognition results as compared to other kernel-based methods. The article of Alba-Castro et al. relates to new concepts in distributed, clientserver-based biometric systems and also user interface aspects. Here, a novel system is presented and evaluated, mainly composed of a client-side face detection and shot selection, whereas pose correction and face recognition is performed on the server. To overcome ambient light effects in mobile face recognition scenarios, Hizem et al. present a concept that can acquire two images at the same time: one in near infrared and the other in visible light, including a novel landmark detection of sufficiently low complexity for application in mobile platforms. They further present an experimental proofof-concept for their system based on three test databases. An important problem in 3-D face recognition is the alignment of facial surfaces. In this area, Salah et al. present a new registration scheme based on multiple average face models including experimental results for applying it to gender and morphology clustering problems, as well as for resampling.

Contributions of this special section to the domain of fingerprint recognition start with two papers on image quality aspects. Theofanos et al. discuss in an experimental study the improvements of fingerprint image quality when involving human computer interaction paradigms, usability considerations, and real-time feedback to the test subject during the acquisition process. Alonso-Fernandez et al. experimentally study fingerprint quality measures in context of different sensor technology (capacitive/optical) and two alternative recognition algorithms (minutiae/ridgebased). Another crucial issue in fingerprint recognition is liveness detection. For this problem, Tan and Schuckers present a new method based on statistical features from noise analysis along the valleys in the ridge-valley structure of fingerprint images, involving wavelet decompositions in multiresolution scales. Based on their experiments, they report significant spoof detection rates. For the authentication of fingerprint templates, Ahmed and Selvanadin suggest an adaptive watermarking scheme, which is based on segmentation of template into regions of interest (ROI) and regions of background (ROB), in order to minimize the degradation of the recognition performance. Authors further present experimental evaluations of their method based on bit error rates for the watermark payload, recognition, and authentication rates.

For the third category of handwriting-based biometric modality, Maiorana et al. introduce a new method for protecting templates for dynamic signature verification: user adaptive fuzzy commitment. It is based on cryptographic techniques and is designed to overcome the possibility to derive original biometrics from stored templates. Their experimental results show the effectiveness of their new method in comparison to nonsecure classification systems, as well as earlier protection schemes. Aging, i.e., the effect of long-term variability of biometric features of individuals to recognition accuracy, constitutes another area of increasing research interest. In this context, Garain et al. present their work on machine dating of hand written manuscripts (i.e., offline handwriting data), which comprises a framework for automatic time stamping based on grapheme clusters, as well as experimental results showing that temporal dating can be achieved to a certain degree.

With respect to multimodal systems, this special section features two papers. First, for the fusion of handwriting and speech in a simultaneous acquisition scenario, Humm et al. present results of their detailed evaluation. Here, the bimodal recognition performance is studied in context of different modeling systems (GMM and HMM), two different training scenarios (MAP and EM), multi-versus mono-session scenarios, as well as skilled versus unskilled forgery attacks. Second, Voloshynovskiy and others motivate the combination of document authentication schemes and multimodal biometrics for an application scenario of high relevance: authentication of biometric documents. Their work consists of a new theoretical framework, practical schemes for robust visual hashing and data hiding, and experimental results for the scenario of mobile devices.

Face, fingerprint, and multiple modalities are certainly topics of high relevance at the time of publication of this journal, due to the large-scale deployment of biometric travel documents. However, the wide variety of alternative physiological and behavioral traits should not be neglected, which are studied by current research. For this special section, we were able to include four papers to represent the variety of those nonmainstream modalities. For iris recognition, Ives et al. address the problem of increasingly large databases by experimentally studying the impact of image compression techniques to recognition results. Uhl and Wild elaborate on a relatively unexplored modality: footprints. They suggest image enhancement and feature extraction steps, emphasizing specific characteristics of foot geometry and their permanence and distinctiveness properties, under consideration of the aspects of collectability and universality. Experimental results are presented comparing discriminative power of foot shape and texture. Shang and Veldhuis focus in their article on a very specific application: rightful user of authentication of a gun based on grip-pattern recognition. The goal of their work is improvement in verification performance of an earlier method by overcoming the variations of pressure distribution and hand position between the probe image and the gallery image of a subject. The authors do so by presenting three areas of improvement in their experimental evaluations: using different sessions for training, image registration, and classifier fusion. Last in this special, but certainly not least in relevance, Dutağacı et al. present a comprehensive experimental study on recent advances in hand-based recognition on a remarkably large database. The study includes comparison of several feature sets in the shape-only and shape-plustexture categories, emphasizes the relevance of a proper hand normalization scheme and preferences with respect to left hand or right hand usage. Also, the experiments have been conducted in consideration of time lapse data and in an open test setup, containing hand images from subjects unseen during the training stage.

At this point, the primary guest editor would like to express his thankfulness to many colleagues, who have 
helped to realize this special section of the Journal of Electronic Imaging. First to mention is Jan Allebach, the editorin-chief of this journal, for giving us the opportunity to publish in this volume and his helpful advise throughout the editorial process. Then, there are of course all the authors who have submitted their work and thus provide the main substance for this special. Furthermore, a particular thank you goes to the co-editors for this special, Berrin Yanikoğlu, Sonia Garcia-Salicetti, Richard M. Guest and Stephen J. Elliott, for their tremendous support in organizing the peer reviews. Also, there have been numerous anonymous reviewers who have volunteered and helped the authors as well as the editors by their valuable comments and expertise. A special thank you goes to the SPIE journal staff, Felicia Andreotta, Rita Davis, and Karolyn Labes, for helping to bear the organizational work for this special section project.

We are confident to have brought together a collection of high-quality papers in relevant areas of recent re- search with a strong affinity to digital image processing. We very much hope that the readers will enjoy reading this special section and that it will have a sustainable impact to the research domain in the intersection of biometrics and digital imaging.

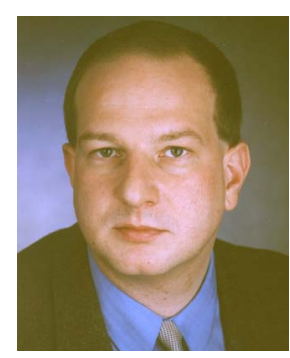

Claus Vielhauer is a professor for IT Security at Brandenburg University of Applied Sciences in Germany and has an additional affiliation as the leader of the biometrics research group as part of the Advanced Multimedia and Security Lab at Otto-von-Guericke University of Magdeburg, Germany. Formerly, he worked for the Multimedia Communications Lab (KOM) of Technical University Darmstadt, Germany, from 1999 to 2004, from where he also received his $\mathrm{MSc}$ and $\mathrm{PhD}$ degrees in electrical engineering. His research interests are in biometrics with specialization in multimodal and behavioral-based recognition, biometric cryptography and applica- tions of biometrics to multimedia, as well as human-to-computer interaction $(\mathrm{HCl})$. Additionally, he is elaborating methods of adopting similarity measurements and classification methods from biometric user authentication to related IT security problems such as digital watermarking, steganography, or IT forensics. He is the author of the book Biometric User Authentication for IT Security: From Fundamentals to Handwriting, published by Springer in 2006, and has a great number of additional book, journal, and conference publications in the areas of biometric signal processing, pattern recognition, $\mathrm{HCl}$, and multimedia security. He has served as guest and associate editor for the Journal of Electronic Imaging and the EURASIP Journal on Information Security and additionally volunteers as a reviewer for renowned journals such as Multimedia Systems, IEEE Transactions on Signal Processing, Pattern Recognition Letters, and Pattern Analysis and Applications. Dr. Vielhauer has co-organized numerous conferences, special sessions, workshops, and tutorials, for example at ACM, IEEE, and SPIE conferences, and has served as a program committee member for many international conferences. 